\title{
HOW IMPORTANT IS THE DARK MATTER HALO FOR BLACK HOLE GROWTH?
}

\author{
Marta Volonteri ${ }^{1}$, Priyamvada Natarajan ${ }^{2,3}$, and Kayhan GÜltekin ${ }^{1}$ \\ ${ }^{1}$ Department of Astronomy, University of Michigan, Ann Arbor, MI, USA \\ ${ }^{2}$ Department of Astronomy, Yale University, New Haven, CT, USA \\ ${ }^{3}$ Institute for Theory and Computation, Harvard-Smithsonian Center for Astrophysics, Cambridge, MA, USA \\ Received 2011 March 8; accepted 2011 May 26; published 2011 July 28
}

\begin{abstract}
In this paper, we examine whether the properties of central black holes in galactic nuclei correlate with their host dark matter halos. We analyze the entire sample of galaxies where black hole mass, velocity dispersion $\sigma$, and asymptotic circular velocity $V_{c}$ have all been measured. We fit $M_{\mathrm{BH}}-\sigma$ and $M_{\mathrm{BH}}-V_{c}$ to a power law, and find that in both relationships the scatter and slope are similar. This model-independent analysis suggests that although the black hole masses are not uniquely determined by dark matter halo mass, when considered for the current sample as a whole, the $M_{\mathrm{BH}}-V_{c}$ correlation may be as strong (or as weak) as $M_{\mathrm{BH}^{-}} \sigma$. Although the data are sparse, there appears to be more scatter in the correlation for both $\sigma$ and $V_{c}$ at the low-mass end. This is not unexpected given our current understanding of galaxy and black hole assembly. In fact, there are several compelling reasons that account for this: (1) supermassive black hole (SMBH) formation is likely less efficient in low-mass galaxies with large angular momentum content, (2) SMBH growth is less efficient in low-mass disk galaxies that have not experienced major mergers, and (3) dynamical effects, such as gravitational recoil, increase scatter preferentially at the low-mass end. Therefore, the recent observational claim of the absence of central SMBHs in bulgeless, low-mass galaxies, or deviations from the correlations defined by high-mass black holes in large galaxies today is, in fact, predicated by current models of black hole growth. We show how this arises as a direct consequence of the coupling between dark matter halos and central black holes at the earliest epochs.
\end{abstract}

Key words: black hole physics - galaxies: evolution - galaxies: formation

Online-only material: color figures

\section{INTRODUCTION}

The demography of local galaxies suggests that most galaxies harbor a quiescent supermassive black hole $(\mathrm{SMBH})$ in their nuclei. Observational evidence also points to the existence of a strong correlation between the masses of the central SMBHs and the properties of the host spheroids (Ferrarese \& Merritt 2000; Gebhardt et al. 2000; Tremaine et al. 2002; Marconi \& Hunt 2003; Häring \& Rix 2004; Gültekin et al. 2009) and possibly the host halos (Ferrarese 2002) in nearby galaxies. These correlations are strongly suggestive of coeval growth of the SMBH and the stellar component likely via regulation of the gas supply in galactic nuclei from the earliest times (Haehnelt et al. 1998; Silk \& Rees 1998; Kauffmann \& Haehnelt 2000; Fabian 2002; King 2003; Thompson et al. 2005; Natarajan \& Treister 2009; Booth \& Schaye 2010).

In a recent pair of papers, Kormendy et al. (2011, KBC hereafter) and Kormendy \& Bender (2011, KB thereafter) have argued that (1) SMBH masses do not appear to correlate with galaxy disks and (2) it is the morphology of the host galaxy that determines whether a correlation with the SMBH exists or not, and the correlation is not contingent on properties of the dark matter halo. In this paper, we re-examine the evidence and demonstrate that the properties of the dark matter halo are, in fact, relevant to the growth and assembly of the central SMBH. Furthermore, we show that correlations between the mass of the SMBH and the host dark matter halo are inevitable during hierarchical growth within the $\Lambda \mathrm{CDM}$ model wherein galaxy assembly is driven by the mergers of dark matter halos (see also Peng 2007; Jahnke \& Maccio 2010). Our goal here is to clarify that some very plausible models for the formation of SMBH seeds at the earliest cosmic epochs do indeed correlate key dark matter halo properties with the masses of the assembling SMBHs. In addition these same models do predict that bulgeless galaxies today are frequently bereft of SMBHs as found by KB (Volonteri et al. 2008; Volonteri \& Natarajan 2009). Our interpretation is that at earlier times the properties of the assembling SMBHs are more tightly coupled to properties of the dark matter halos as their growth is driven by the merger history of halos. However, at later times the final mass of the SMBH becomes more tightly coupled to the small-scale local baryonic distribution.

\section{BLACK HOLES AND BULGES}

In their recent analysis of correlations between black holes and dark matter halos, KB found that halo mass (measured with circular velocity, $V_{c}$ ) was a far poorer predictor of black hole mass compared to, e.g., galaxy stellar velocity dispersion $(\sigma)$ or bulge luminosity $\left(L_{\text {bulge }}\right)$, especially for smaller galaxies. This trend is further displayed by an apparent breakdown in small galaxies of the otherwise tight, linear correlation between $V_{c}$ and $\sigma$. A linear correlation is expected for simple galaxy models so that the observed deviation in small galaxies is argued to imply no correlation. KBC interpret the tight, linear correlation between $V_{c}$ and $\sigma$ in large galaxies arising from the well-known "cosmic conspiracy."

As per KB it does appear that today $M_{\mathrm{BH}}$ and $V_{c}$ are, in fact, correlated but possibly more weakly so, particularly at lower masses, compared to the $M_{\mathrm{BH}}-\sigma$ and $M_{\mathrm{BH}}-L_{\text {bulge }}$ relations. However, we offer an alternative interpretation for why this is so. We argue here that this is likely a direct consequence of the role of merging dark matter halos that drive SMBH growth. Our view hinges on the fact that the coupling between SMBH growth and dark matter was necessarily strong at high redshifts 


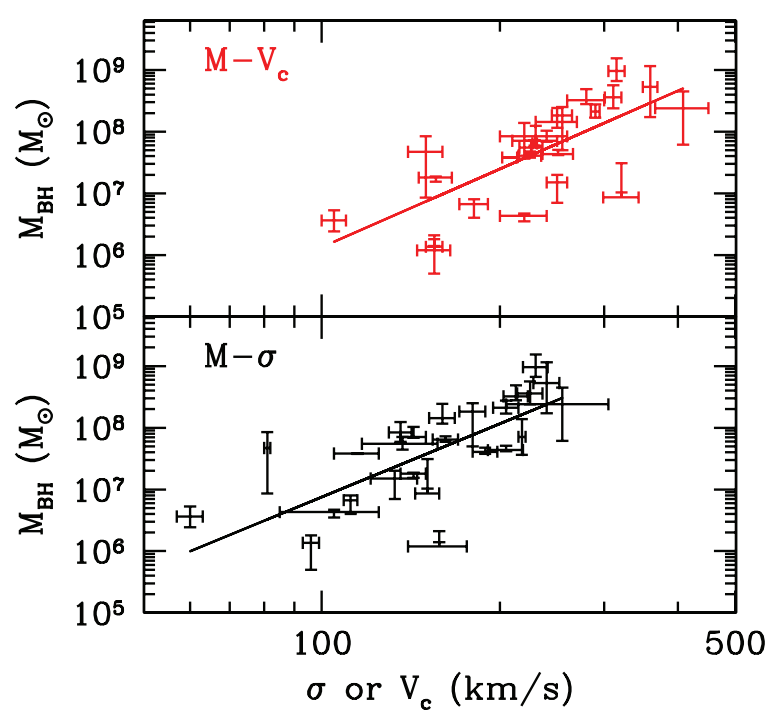

Figure 1. Top panel: $M_{\mathrm{BH}}-V_{c}$ relation for the 25 galaxies in Table 1 of KBC. Bottom panel: $M_{\mathrm{BH}}-\sigma$ relation from Kormendy's table. The best fit in each case is also shown.

(A color version of this figure is available in the online journal.)

as merger rates determine the assembly history, and major mergers that trigger accretion episodes are more frequent at high redshift for the most massive halos. At late times, however, the SMBH mass itself is more tightly coupled to the properties of the baryonic galactic nucleus, in particular, for low-mass galaxies that have experienced practically no major mergers in their entire lifetime. The key point here is that major mergers trigger simultaneous SMBH growth and star formation causing a tight coupling between these two components in the galactic nucleus only for massive halos.

To explore this interpretation further, we first re-examine the data from $\mathrm{KBC}$ from a purely empirical perspective. The sample is composed of 25 galaxies with dynamical measurements of the black hole mass, $M_{\mathrm{BH}}$, and high-quality measurements of velocity dispersion, $\sigma$, and asymptotic circular velocity, $V_{c}$ (Figure 1). Galaxy properties are listed in Table 1 of the supplementary information in KBC. We fit the data with a functional form of

$$
\log _{10}\left(\frac{M_{\mathrm{BH}}}{M_{\odot}}\right)=A+B \log _{10}\left(\frac{V_{c}}{200 \mathrm{~km} \mathrm{~s}^{-1}}\right) .
$$

Using a symmetric least-squares fit, we find $A=7.2 \pm 0.05$ and $B=7.60 \pm 0.40$ with $\chi^{2} / \mathrm{dof}=7.2$, indicating that the data are very unlikely to have come from the model used, in strong agreement with $\mathrm{KB}$.

We then expand the model to include a log-normal scatter about the relation of standard deviation $s_{0}$. The inclusion of a scatter term of some sort is essential since the deviations from a log-linear relation are in excess of the measurement errors (Hogg et al. 2010). Using the methods of Gültekin et al. (2009), we fit a generalized maximum likelihood method that can handle measurement errors in the independent and dependent variables (assumed Gaussian in log space) as well as upper limits. We find $A=7.39 \pm 0.14, B=4.22 \pm 0.93$, and $s_{0}=0.53 \pm 0.10$, which indicates that a correlation can be inferred from the data. Compared to the $M_{\mathrm{BH}}-\sigma$ relation, the scatter in the $M_{\mathrm{BH}}-V_{c}$ relation is (1) slightly larger for this sample, for which we find $A=8.06 \pm 0.14, B=3.95 \pm 0.72$, and $s_{0}=0.50 \pm 0.09$; (2) significantly larger than the entire sample in Gültekin et al.
(2009) $\left(s_{0}=0.44 \pm 0.06\right)$; and (3) much larger than the ellipticalonly sample in Gültekin et al. (2009) $\left(s_{0}=0.31 \pm 0.06\right)$.

Given that the sample was selected based on the ability to measure $V_{c}$ in each galaxy, the actual scatter in the $M_{\mathrm{BH}}-V_{c}$ relation may be even larger since those galaxies in which $V_{c}$ is difficult to measure will tend to be outliers. The fact that the inferred scatter in the $M_{\mathrm{BH}}-V_{c}$ relation is not smaller than the scatter in the $M_{\mathrm{BH}}-\sigma$ implies that the halo mass is not driving the correlation at a higher level than the physical process that sets the bulge properties. This first assessment corroborates one of the main conclusions of KB that a central black hole's mass today is not uniquely determined by the mass of the dark matter halo. The similar level of scatter in the $M_{\mathrm{BH}}-\sigma$ and $M_{\mathrm{BH}}-V_{c}$ fits, however, confirms that there is a trend in the entire $M_{\mathrm{BH}}-V_{c}$ sample (plus notable outliers; see Figure 1. For example, there is only one galaxy with $V_{c}>250 \mathrm{~km} \mathrm{~s}^{-1}$ and $M_{\mathrm{BH}}<10^{8} M_{\odot}$ and only one with $V_{c}<200 \mathrm{~km} \mathrm{~s}^{-1}$ and $M_{\mathrm{BH}}>2 \times 10^{7} M_{\odot}$. It is also possible to interpret the data as having only a weak correlation below $M_{\mathrm{BH}}<5 \times 10^{7} M_{\odot}$ ). We show below how this trend is expected to naturally arise in a set of physically well-motivated models for the formation of SMBH seeds at the earliest epochs without requiring black holes to partake in exotic nonbaryonic physics.

\section{LINKS BETWEEN SMBH FORMATION, HALO MASS, AND SPIN}

We focus on a class of SMBH seeding models to illustrate that the observed lack of central black holes in low-velocity dispersion bulgeless galaxies today need not imply a lack of correlation between SMBH and dark matter halo properties at earlier epochs (Volonteri et al. 2008; Volonteri \& Natarajan 2009). We describe below a class of black hole seed formation models where the seed properties are initially correlated to the host dark matter properties at high redshifts. Evolving such models via the merger-driven accretion prescription over cosmic time, Volonteri \& Natarajan (2009) find that a key prediction is that low-mass bulgeless galaxies today are unlikely to host nuclear black holes. The relevant host dark matter halo property in this picture is the spin. In a physically motivated model for the formation of heavy SMBH seeds (in contrast to the lower mass remnant seeds from Population III stars) according to the prescription described in Lodato \& Natarajan (2006, 2007) there is a limited range of halo spins and halo masses that are viable sites for the formation of seeds. Details of these models have been presented in several published papers and here we merely paraphrase the relevant details.

In direct collapse models, SMBH seeds can form at high redshift $(z>15)$ in metal-free galaxies with $T_{\text {vir }}>$ $10^{4} \mathrm{~K}$, where atomic hydrogen cooling becomes effective (Koushiappas et al. 2004; Begelman et al. 2006; Lodato \& Natarajan 2006, 2007). Here we refer to Lodato \& Natarajan $(2006,2007)$ for more details of the seeding model wherein the development of non-axisymmetric spiral structures drives mass infall and accumulation in a pre-galactic disk with primordial composition. The mass accumulated in the center of the halo (which provides an upper limit to the SMBH seed mass) is given by

$$
M_{\mathrm{BH}}=m_{\mathrm{d}} M_{h}\left[1-\sqrt{\frac{8 \lambda}{m_{\mathrm{d}} Q_{\mathrm{c}}}\left(\frac{j_{\mathrm{d}}}{m_{\mathrm{d}}}\right)\left(\frac{T_{\mathrm{gas}}}{T_{\mathrm{vir}}}\right)^{1 / 2}}\right] \text {, }
$$




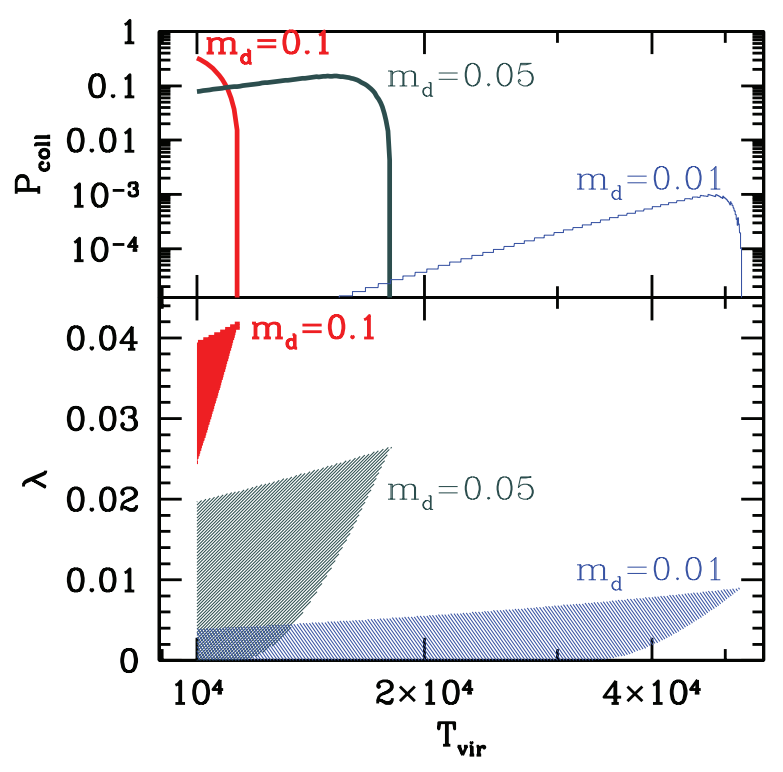

Figure 2. Parameter space (virial temperature and spin parameter) for SMBH formation. Here we select halos with $T_{\mathrm{vir}}>10^{4} \mathrm{~K}$ at $z=15$ for different gas fractions that participate in the infall $\left(m_{\mathrm{d}}\right)$. Other adopted parameters are $m_{\mathrm{d}}=j_{\mathrm{d}}, \alpha_{\mathrm{c}}=0.06$, and here we consider the $Q_{\mathrm{c}}=2$ case (cf. Volonteri et al. 2008). The gas has a temperature $T_{\text {gas }}=5000 \mathrm{~K}$. The shaded areas in the bottom panel show the range of virial temperatures and spin parameters where disks are Toomre unstable and the joint conditions, $\lambda<\lambda_{\max }$ (Equation 3 ) and $T_{\text {vir }}<T_{\max }$ (Equation 4, providing the minimum spin parameter, $\lambda_{\min }$ value below which the disk is globally prone to fragmentation) are fulfilled. The top panel shows the probability of SMBH formation, by integrating the log-normal distribution of spin parameters between $\lambda_{\min }$ and $\lambda_{\max }$.

(A color version of this figure is available in the online journal.)

for

$$
\lambda<\lambda_{\max }=m_{\mathrm{d}} Q_{\mathrm{c}} / 8\left(m_{\mathrm{d}} / j_{\mathrm{d}}\right)\left(T_{\mathrm{vir}} / T_{\mathrm{gas}}\right)^{1 / 2},
$$

and $M_{\mathrm{BH}}=0$ otherwise. Here $\lambda_{\max }$ is the maximum halo spin parameter for which the disk is gravitationally unstable, $m_{d}$ is the gas fraction that participates in the infall, $j_{\mathrm{d}}$ is its specific angular momentum, and $Q_{\mathrm{c}} \gtrsim 1$ is the Toomre parameter. The efficiency of the seed assembly process ceases at large halo masses, where the disk undergoes fragmentation instead. This occurs when the virial temperature exceeds a critical value $T_{\max }$, given by

$$
\frac{T_{\max }}{T_{\mathrm{gas}}}=\left(\frac{4 \alpha_{\mathrm{c}}}{m_{\mathrm{d}}} \frac{1}{1+M_{\mathrm{BH}} / m_{\mathrm{d}} M_{h}}\right)^{2 / 3},
$$

where $\alpha_{\mathrm{c}} \approx 0.06$ is a dimensionless parameter measuring the critical gravitational torque above which the disk fragments (Rice et al. 2005).

To summarize, a dark matter halo is characterized by its mass $M_{h}$ (or virial temperature $T_{\mathrm{vir}}$ ) and by its spin parameter $\lambda$. If $\lambda_{\min }<\lambda<\lambda_{\max }$ (see Equations (3) and (4)) and $10^{4} \mathrm{~K}<$ $T_{\mathrm{vir}}<T_{\max }$ (Equation (4)), then a seed SMBH can form in the center. Hence, SMBHs form (1) only in halos within a given range of virial temperatures, hence, halo masses, and (2) only within a narrow range of spin parameters, as shown in Figure 2. Low-mass halos $\left(T_{\text {vir }}<10^{4} \mathrm{~K}\right)$ and halos with high values of $\lambda$, most likely leading to disk-dominated galaxies, are strongly disfavored as SMBH seed sites in this model or in others that rely on global dynamical instabilities (see Begelman et al. 2006; Volonteri \& Begelman 2010). Therefore, these models of SMBH formation naturally predict that bulgeless galaxies are unlikely to host SMBHs today, in agreement with KBC's conclusions.

\section{LINK BETWEEN SMBH GROWTH AND HALO MASS}

We briefly outline familiar aspects of the mass assembly history of black holes. Details and discussion on the relevant literature and background can be found in Volonteri \& Natarajan (2009), and references therein.

Accretion episodes are triggered by major mergers, and they drive the mass growth of the central black holes. These very major mergers are also implicated in the formation of bulges. In the $\Lambda \mathrm{CDM}$ framework, the lowest mass halos, though, experience mostly minor mergers, which do not trigger accretion episodes and hence do not grow the SMBH or form a bulge. Effectively, for the lowest mass halos, growth of the stellar component of the galaxy and the central SMBH are not coeval, given that major mergers are the key drivers. Even if these low-mass halos are initially seeded we predict that most low-surface brightness, low-mass bulgeless galaxies at the present time do not host central black holes (Volonteri \& Natarajan 2009). In galaxies that are now high mass, SMBH growth and assembly of the stellar component most likely proceeded coevally over cosmic history. Regulation of the gas physics that underlies the evolution of both processes occurred in the inner regions of the galaxies, triggered by galaxy mergers.

In currently successful theoretical models that fit observational data of accreting black holes over a range of redshifts, the growth of an SMBH is modulated by the history of its host, which in turn depends on a merger history that is set by the properties of the dark matter halo. The host dark matter halo and its merger properties in the context of $\Lambda \mathrm{CDM}$ are crucial to black hole growth at early epochs. The results and predictions of these models have been presented in detail in several papers as mentioned above and there is a fair degree of consensus among the community that these analytic Monte Carlo mergertree-based models do adequately capture most of the relevant physics.

In order to clarify the relevant aspects of these models for our discussion here, we now explicitly develop an analytical model that uses only halo merger rates to delineate the role of dark matter halos for black hole growth. As an ansatz, we start with the premise that SMBHs grow "healthily" only if their host experiences a major merger. Furthermore, we assume major mergers bring an SMBH on the scaling relations expected for their hosts, for instance via self-regulation and active galactic nucleus (AGN) feedback (e.g., Silk \& Rees 1998; Fabian 1999; Di Matteo et al. 2005). The details of these so-called AGN feedback processes are unsettled at the present time, as there is no entirely convincing theoretical model, or simulation for that matter, wherein the detailed microphysics of these feedback processes is captured. For our purposes, however, it is sufficient to simply invoke them as a means to setting up the scaling relations. The existence of these scaling relations suggests that such processes ought to occur.

Here, we use just the halo merger rate as the starting point for evolving black hole masses over time. High-resolution dark matter only cosmological simulations provide us the merger rates for halos, e.g., Fakhouri et al. (2010). In fact, a simple fitting formula can be derived (Equation (1) in Fakhouri et al. 2010) for the merger rate per unit redshift and mass ratio $(\xi \leqslant 1)$ 
at fixed halo mass ${ }^{4}$ :

$$
\frac{d N_{m}}{d \xi d z}\left(M_{h}\right)=A_{0}\left(\frac{M_{h}}{10^{12} M_{0}}\right)^{\alpha} \xi^{\beta} \exp \left[\left(\frac{\xi}{\tilde{\xi}}\right)^{\gamma}\right](1+z)^{\eta}
$$

with $A_{0}=0.0104, \alpha=0.133, \beta=-1.995, \gamma=0.263$, $\eta=0.0993$, and $\tilde{\xi}=9.72 \times 10^{-3}$. We integrate the merger rate between $z=0$ and $z=3$, and mass ratio $\xi>0.3$ (major mergers. The minimum mass ratio that triggers growth episodes for the central black hole can be as low as $\xi>0.1$, however for the successful formation of bulges the threshold needs to be raised, e.g., Somerville et al. 2001). This gives the number of major mergers a halo of a given mass experiences between $z=0$ and $z=3$. These major mergers are expected to be responsible for the simultaneous growth of bulges and SMBHS (e.g., Di Matteo et al. 2005). If a halo experiences at least one major merger, then its SMBH has a chance of growing in a self-regulated manner (via AGN feedback, as mentioned above) with its host. If a halo does not experience any major merger, then the growth of the SMBH is expected to be decoupled from the properties of the host, being driven by, e.g., secular effects (cf. KBC).

It is apparent from Equation (5) that the major merger rate is an increasing function of halo mass/circular velocity. In fact, we find that the expected number of mergers between $z=0$ and $z=3$ with mass ratio $\xi>0.3$ is approximately 0.4 for $M_{h}=10^{8} M_{\odot}, 0.5$ for $M_{h}=10^{9} M_{\odot}, 0.7$ for $M_{h}=10^{10} M_{\odot}$, and it is above unity only for $M_{h}>10^{11} M_{\odot}$. Therefore, if the correlation between SMBH masses and hosts is established by the simultaneous growth of the bulge and SMBH triggered by major mergers, SMBHs in low-mass galaxies are simply more likely to be outliers. Here, we have shown clearly the role that major mergers play in the triggering of mass growth for central black holes and that it is the dark matter halo mass that is the key determinant for deriving the major merger rate. Deviations from the scaling relations that are a direct consequence of this merger-driven scenario are therefore expected and inevitable for low-mass halos. Below we show explicitly how these deviations are likely set up for low-mass halos/galaxies and how these are a result of their growth histories.

To quantify deviations from the $M_{\mathrm{BH}}-V_{c}$ relation, we run a Monte Carlo simulation of the growth of 560,000 galaxies, and ask at $z=0$ how many SMBHs are "ungrown" (no major mergers), "ejected" (kicked because of gravitational recoil), or "healthy" (at least one major merger brought the SMBH on the correlation, and if an SMBH has been ejected, a major merger after the ejection is required to bring the SMBH back onto the $M_{\mathrm{BH}}-V_{c}$ relation).

We create a Monte Carlo realization of halo mergers per halo as a function of mass, where the probability of a major merger and the mass ratio of a given major merger are drawn from the distribution defined in Equation (6). We first assume that initially each halo hosts an SMBH and that its mass is predicted by the $M_{\mathrm{BH}}-V_{c}$ relation that is proportional to the halo mass to the $4 / 3$ power (combining the relationships $M_{\mathrm{BH}}-V_{c}$ and $M_{h}-V_{c}$ ). From the mass ratio of merging halos $(\xi)$ we can derive the mass ratio

4 Halo mass can be translated into virial circular velocity

$$
V_{\mathrm{c}}=142 \mathrm{~km} \mathrm{~s}^{-1}\left[\frac{M_{h}}{10^{12} M_{\odot}}\right]^{1 / 3}\left[\frac{\Omega_{m}}{\Omega_{m}^{z}} \frac{\Delta_{\mathrm{c}}}{18 \pi^{2}}\right]^{1 / 6}(1+z)^{1 / 2},
$$

where $\Delta_{\mathrm{c}}$ is the overdensity relative to the critical density. For a WMAP5 cosmology we adopt the fitting formula $\Delta_{\mathrm{c}}=18 \pi^{2}+82 d-39 d^{2}$ (Bryan \& Norman 1998), where $d \equiv-1+\Omega_{m}(1+z)^{3} /\left(\Omega_{m}(1+z)^{3}+\Omega_{\Lambda}+\Omega_{k}(1+z)^{2}\right)$.

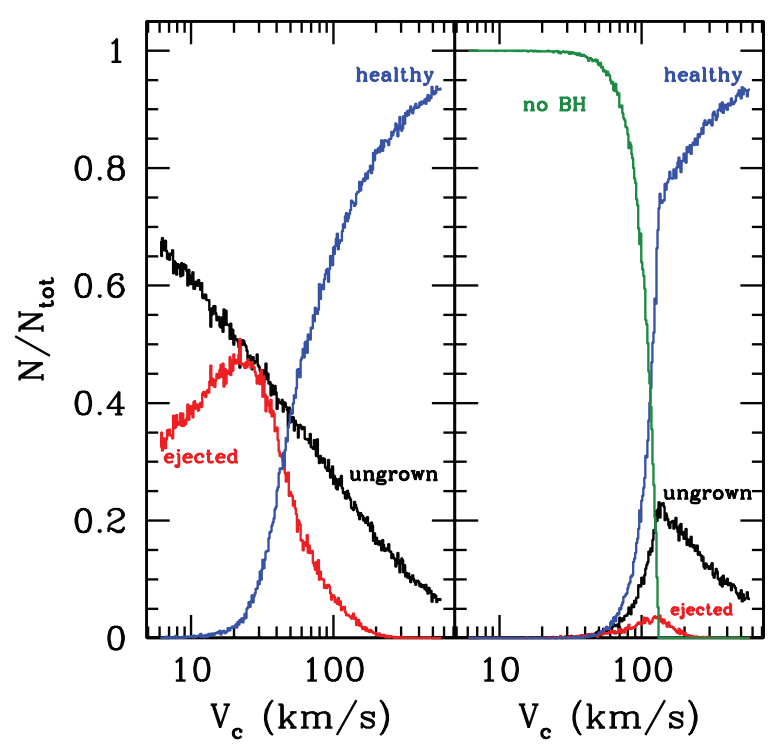

Figure 3. Distribution of SMBH growth histories as a function of halo mass. If an SMBH is hosted in a galaxy that has not experienced a major merger $(\xi>0.3)$ since $z=3$ we dub it "ungrown." If the galaxy experiences a major merger that results in the SMBH being ejected, and no further major merger brings in $\mathrm{SMBH}$, we assume the galaxy has no SMBH ("ejected"). A "healthy" SMBH is likely to sit on or near the expected $M_{\mathrm{BH}}-V_{c}$ relation. "Healthy" SMBHs are hosted in galaxies where at least one major merger brought the SMBH on the correlation, and if an SMBH has been ejected, we require a major merger after the fact to bring in a new SMBH and grow it onto the $M_{\mathrm{BH}}-V_{c}$ relation. The left panel assumes that all halos host an SMBH, regardless of mass. The right panel assumes an "occupation fraction" of SMBHs derived from Figure 4 in $\operatorname{VLN} 2008\left(Q_{c}=2\right)$.

(A color version of this figure is available in the online journal.)

of merging SMBHs ( $q=\xi^{4 / 3}$ ). We further assume a probability distribution of SMBH spins (we assumed a random distribution between 0 and 1), and of inclination between SMBH spins and orbital angular momentum at the time of SMBH merger (we assumed partial alignment, cf. the "hot" case in Dotti et al. 2010; Volonteri et al. 2010b, results are qualitatively insensitive to this choice) and derive gravitational recoil velocities (van Meter et al. 2010) for each SMBH merger in our sample. By comparing these recoil velocities to escape velocities from halos, assumed to be described by Navarro-Frenk-White profiles, we can derive an ejection probability (see Volonteri et al. 2010b). We then run a second realization where we assume an SMBH "occupation fraction" (OF, i.e., the fraction of halos that hosts SMBHs) derived from Figure 4 in VLN2008 $\left(Q_{c}=2\right)$. Using a linear fit in log-space, the occupation fraction scales with the halo mass as

$$
\mathrm{OF}=\min \left(10^{1.3 \log \left(M_{\mathrm{h}} / 10^{12} M_{\odot}\right)}, 1\right) .
$$

Figure 3 shows the distribution of SMBH growth histories. It is apparent that most SMBHs in galaxies with $V_{c} \gtrsim 100 \mathrm{~km} \mathrm{~s}^{-1}$ have experienced at least one major merger between $z=3$ and $z=0$. Given the assumption that major merger-triggered accretion brings SMBHs on or near the expected $M_{\mathrm{BH}}-V_{c}$ relation, these SMBHs are "healthy."

The fraction of outliers increases as we move toward lower and lower masses, where most SMBHs are either ungrown or ejected, or where the galaxy never hosted an SMBH in the first place. We note that the effect of gravitational recoil (ejections) is strongest at intermediate galaxy masses. This is because lowmass galaxies have few mergers while high-mass galaxies have large escape velocities. Since low-mass galaxies have a small 


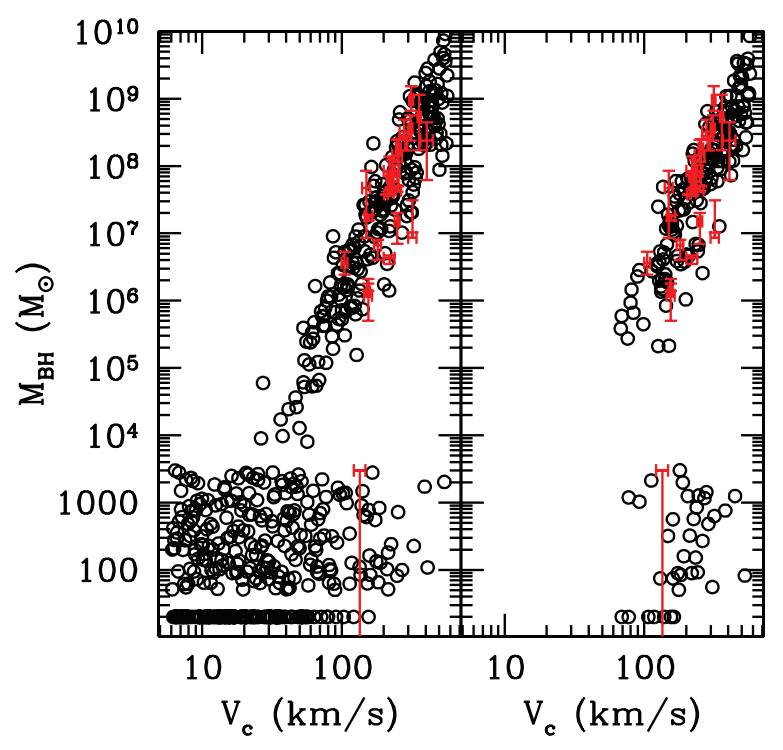

Figure 4. Circles: $M_{\mathrm{BH}}-V_{c}$ relation from our Monte Carlo simulations. Here at every major merger an SMBH increases its mass by $10^{8} M_{\odot}\left(V_{\mathrm{c}} / 350 \mathrm{~km} \mathrm{~s}^{-1}\right)^{4} \times$ $10^{0.5 \Delta}$ where $\Delta$ is normally distributed about 0 with standard deviation 1 . The mass of "ungrown" SMBHs is set to $300 M_{\odot} \times 10^{2 R-1}$, where $R$ is randomly distributed between 0 and 1 . Ejected SMBHs are arbitrarily set to $M_{\mathrm{BH}}=10 M_{\odot}$ to show which galaxies are affected. The left panel assumes that all halos host an SMBH, regardless of mass. The right panel assumes an "occupation fraction" of SMBHs derived from Figure 4 in VLN2008 $\left(Q_{c}=2\right)$. Galaxies from KBC are shown with error bars. M33 is shown as the upper limit at $3000 M_{\odot}$.

(A color version of this figure is available in the online journal.)

number of major mergers, or they have no $\mathrm{SMBH}$, even if the ejection probability-based on the comparison between the escape and recoil velocities-is close to $100 \%$, if a galaxy has no major merger at all, then the ejection probability convolved with the merger probability is zero.

This analytical model therefore predicts the halo mass where the transition from healthy growth (triggered by major mergers) to unhealthy SMBHs (in the sense that their masses are not set by merger-driven accretion) occurs. One can integrate Equation (5) at different redshifts to find where this transition occurs at early times, if desired. The transition corresponds to the halo mass (or circular velocity) where the average number of major mergers drops below unity.

In Figure 4 , we present the $M_{\mathrm{BH}}-V_{c}$ relation for 560 random galaxies in our Monte Carlo sample (uniformly drawn out of the complete sample of 560,000) and compare it to the $M_{\mathrm{BH}}-V_{c}$ of the 25 galaxies described in Section 2 (from Table 1 in KBC, augmented by the upper limit for M33). Here we assume that at every major merger an SMBH increases its mass by $10^{8} M_{\odot}\left(V_{\mathrm{c}} / 350 \mathrm{~km} \mathrm{~s}^{-1}\right)^{4} \times 10^{0.5 \Delta}$ where $\Delta$ is normally distributed about 0 with standard deviation 1 (see Section 2 for details). These are "healthy" SMBHs. The mass of "ungrown" SMBHs is set to $300 M_{\odot} \times 10^{2 R-1}$, where $R$ is randomly distributed between 0 and 1 . "Ejected" SMBHs are arbitrarily set to $M_{\mathrm{BH}}=10 M_{\odot}$ to demarcate the affected galaxies. The Monte Carlo sample clearly occupies the same area as occupied by the real galaxies. Therefore, the deviations from the scaling relation at low masses today reflect the growth history of black holes that is driven principally by the dark matter halo mass.

\section{DISCUSSION AND CONCLUSIONS}

Analyzing the entire sample of galaxies where black hole mass, velocity dispersion $\sigma$, and asymptotic circular velocity $V_{c}$ have all been measured, we obtain the best-fit power-law relation between $M_{\mathrm{BH}}-\sigma$ and $M_{\mathrm{BH}}-V_{c}$, and find that the scatter and slope are very similar for both relations. This model-independent fit suggests that the $M_{\mathrm{BH}}-V_{c}$ correlation is just as strong (or just as weak) as the correlation between $M_{\mathrm{BH}}-\sigma$ given current sample sizes. As noted by KB, the correlations worsen (or disappears) for $\sigma$ and $V_{c}$ outside a $180-260 \mathrm{~km} \mathrm{~s}^{-1}$ range.

In this paper, we have argued that in the context of our current understanding of the growth of galaxies and black hole assembly, this is not unexpected. Most importantly, the absence of central SMBHs in bulgeless low-mass galaxies today is not necessarily an indication of the lack of correlation between the dark matter halo and the central object at every epoch. In fact, it is a consequence of the weaker coupling of the dark matter halo with central black holes for low-mass halos that experience practically no major mergers in their lifetime. With an explicit example we show that black hole seed models at high redshift that do assume a strong coupling between SMBH seed masses and the spin of the dark matter halo, do predict that low-mass bulgeless galaxies today should be bereft of central black holes. The key reason for this late-time consequence is that SMBH formation is less efficient in low-mass galaxies with large angular momentum and these seeds are also less likely to grow.

Additionally, the growth of SMBHs in low-mass galaxies is hampered, as such galaxies rarely experience major mergers. In particular, as suggested by KBC secular effects that can build pseudo-bulges might at late times decouple the properties of the central stellar-dominated region from the overall dark matter halo. Besides, dynamical effects, such as gravitational recoil and ejection, increase deviations preferentially at $V_{c}$ below $\sim 100-200 \mathrm{~km} \mathrm{~s}^{-1}$. Therefore, we propose that dark matter halos do drive the overall formation and growth of black holes, in the sense that they set the stage for SMBH growth through the merger history of the host, set by large-scale structure. However, especially for low-mass galaxies where the star formation history and SMBH growth are not driven by major mergers, we do not expect strong correlations between $\sigma$ (set by the stellar component) or $M_{\mathrm{BH}}$ (set by baryonic processes such as accretion of gas released by stellar winds, see Volonteri et al. 2010a) and $V_{c}$ (set by the dark matter halo) today.

On the issue of central SMBHs in cluster galaxies, we note that tidal stripping strongly truncates the dark matter distribution of galaxies infalling into clusters, while leaving the inner regions that are primarily baryonic unaltered (see Natarajan \& Treister 2009). Besides, it appears that tidal stripping is more efficient for late-type galaxies. Therefore, the relation between $\sigma$ and $V_{c}$ for late-type cluster galaxies (more so than for early-types) will not be the same as for equivalent luminosity field galaxies. Regardless of which is the more fundamental correlation, $M_{\mathrm{BH}}-\sigma$ or $M_{\mathrm{BH}}-V_{c}$, these correlations are likely to differ more significantly for cluster galaxies.

P.N. thanks the John Simon Guggenheim Foundation for the award of a Guggenheim fellowship and the Institute for Theory and Computation at the Harvard Smithsonian Center for Astrophysics for hosting her. M.V. acknowledges support from SAO Award TM1-12007X and NASA awards ATP NNX10AC84G and NNX07AH22G.

\section{REFERENCES}

Begelman, M. C., Volonteri, M., \& Rees, M. J. 2006, MNRAS, 370, 289 Booth, C. M., \& Schaye, J. 2010, MNRAS, 405, L1 
Bryan, G. L., \& Norman, M. L. 1998, ApJ, 495, 80

Di Matteo, T., Springel, V., \& Hernquist, L. 2005, Nature, 433, 604

Dotti, M., Volonteri, M., Perego, A., Colpi, M., Ruszkowski, M., \& Haardt, F. 2010, MNRAS, 402, 682

Fabian, A. C. 1999, MNRAS, 308, L39

Fabian, A. C. 2002, in ASP Conf. Ser. 258, Issues in Unification of Active Galactic Nuclei, ed. R. Maiolino, A. Marconi, \& N. Nagar (San Francisco, CA: ASP), 185

Fakhouri, O., Ma, C., \& Boylan-Kolchin, M. 2010, MNRAS, 406, 2267

Ferrarese, L. 2002, ApJ, 572, 90

Ferrarese, L., \& Merritt, D. 2000, ApJ, 539, L9

Gebhardt, K., et al. 2000, ApJ, 539, L13

Gültekin, K., et al. 2009, ApJ, 698, 198

Haehnelt, M., Natarajan, P., \& Rees, M. J. 1998, MNRAS, 300, 817

Häring, N., \& Rix, H. W. 2004, ApJ, 604, L89

Hogg, D. W., Bovy, J., \& Lang, D. 2010, arXiv:1008.4686

Jahnke, K., \& Maccio, A. 2010, ApJ, 734, 92

Kauffmann, G., \& Haehnelt, M. 2000, MNRAS, 311, 576

King, A. 2003, ApJ, 596, L27

Kormendy, J., \& Bender, R. 2011, Nature, 469, 377 (KB)
Kormendy, J., Bender, R., \& Cornell, M. E. 2011, Nature, 469, 374 (KBC)

Koushiappas, S. M., Bullock, J. S., \& Dekel, A. 2004, MNRAS, 354, 292

Lodato, G., \& Natarajan, P. 2006, MNRAS, 371, 1813

Lodato, G., \& Natarajan, P. 2007, MNRAS, 377, L64

Marconi, A., \& Hunt, L. 2003, ApJ, 598, L21

Natarajan, P., \& Treister, E. 2009, MNRAS, 393, 838

Peng, C. Y. 2007, ApJ, 671, 1098

Rice, W. K. M., Lodato, G., \& Armitage, P. J. 2005, MNRAS, 364, L56

Silk, J., \& Rees, M. J. 1998, A\&A, 331, L1

Somerville, R. S., Primack, J. R., \& Faber, S. M. 2001, MNRAS, 320, 504

Thompson, T., Murray, J., \& Quaetert, E. 2005, ApJ, 630, 161

Tremaine, S., et al. 2002, ApJ, 574, 740

van Meter, J. R., Miller, M. C., Baker, J. G., Boggs, W. D., \& Kelly, B. J. 2010, ApJ, 719, 1427

Volonteri, M., \& Begelman, M. C. 2010, MNRAS, 409, 1022

Volonteri, M., Dotti, M., Campbell, D., \& Mateo, M. 2010a, ApJ, 730, 145

Volonteri, M., Gültekin, K., \& Dotti, M. 2010b, MNRAS, 404, 2143

Volonteri, M., Lodato, G., \& Natarajan, P. 2008, MNRAS, 383, 1079

Volonteri, M., \& Natarajan, P. 2009, MNRAS, 400, 1911 